\title{
1 Synchronous seasonality in the gut microbiota of wild wood mouse
}

\section{2 populations}

3 Marsh K. J. ${ }^{* 1,4}$, Raulo A. M. ${ }^{2}$, Brouard, M. ${ }^{2}$, Troitsky, T. $^{2}$, English, H. M. ${ }^{1,2}$, Allen, B. ${ }^{3}$, Raval, R. ${ }^{3}$,

4 Webster J. P. ${ }^{1} \&$ Knowles S. C. L. ${ }^{1,2}$

$5 \quad{ }^{1}$ Department of Pathobiology and Population Sciences, The Royal Veterinary College,

6 University of London, Hawkshead Campus, Hatfield, Hertfordshire, AL9 7TA

$7 \quad{ }^{2}$ Department of Zoology, University of Oxford, Mansfield Road, Oxford, Oxfordshire, OX1 3SZ

$8{ }^{3}$ Department of Life Sciences, Imperial College London, Silwood Park, Buckhurst Road, Ascot,

9 SL5 7PY

$10{ }^{4}$ College of Life and Environmental Sciences, University of Exeter, Penryn Campus, Cornwall,

11 UK

12

13 Author for correspondence*:

14 Kirsty J Marsh

15 College of Life and Environmental Sciences

16 University of Exeter

17 Penryn Campus

18 Cornwall, UK

19 TR10 9FE

20 Email: k.j.marsh@exeter.ac.uk 


\section{Abstract}

1. The gut microbiome performs many important functions in mammalian hosts, with community composition shaping its functional role. However, what factors drive individual microbiota variation in wild animals and to what extent these are predictable or idiosyncratic across populations remains poorly understood.

2. Here, we use a multi-population dataset from a common rodent species (the wood mouse, Apodemus sy/vaticus), to test whether a consistent set of 'core' gut microbes is identifiable in this species, and to what extent the predictors of microbiota variation are consistent across populations.

3. Between 2014 and 2018 we used capture-mark-recapture and 165 rRNA profiling to intensively monitor two wild UK mouse populations and their gut microbiota, as well as characterising the microbiota from a laboratory-housed colony of the same species.

4. Although broadly similar at high taxonomic levels and despite being only $50 \mathrm{~km}$ apart, the two wild populations did not share a single bacterial amplicon sequence variant (ASV). Meanwhile, the laboratory-housed colony shared many ASVs with one of the wild populations from which it is thought to have been founded decades ago. Despite strong taxonomic divergence in the microbiota, the factors predicting compositional variation in each wild population were remarkably similar. We identified a strong and consistent pattern of seasonal microbiota restructuring that occurred at both sites, in all years, and within individual mice. While the microbiota was highly individualised, seasonal convergence in the gut microbiota among individuals occurred in late winter/early spring.

5. These findings reveal highly repeatable seasonal gut microbiota dynamics across distinct populations of this species, despite divergent taxa being involved. Providing a platform for future work to understand the drivers and functional implications of such 

host with adaptive seasonal phenotypic plasticity.

51 Keywords: Bacteroidales, core, individuality, lab vs wild, microbiome, mouse, seasonality, 16S

\section{Introduction}

54 The gastrointestinal tracts of vertebrates harbour complex microbial communities known as

55 the gut microbiota, that can perform a wide range of important functions for the host. These

56 include regulating the immune system (Round \& Mazmanian, 2009), extracting nutrients from

57 otherwise indigestible parts of the diet (Flint et al., 2012), and defence against pathogens

58 (Buffie \& Pamer, 2013). With such important roles, one might expect these symbiotic

59 communities to be under strong host influence, such that individuals of a given species

60 harbour a characteristic and relatively invariant community. Yet the emerging picture from

61 microbiome research firmly contradicts this. Vertebrate gut microbiotas are typified by

62 immense compositional variation, both among individuals and within individuals over time.

63 Each individual's gut microbiota constitutes a diverse community of microbes shaped by

64 environmental influences such as diet, habitat and xenobiotics, microbial interactions,

65 metacommunity-level processes such as transmission among hosts, and mechanisms of host

66 selection, arising for example through physiological differences across host genotypes or age.

67 The relative importance of these different ecological processes in shaping wild animal

68 microbiomes remains a key open question.

69 In humans, cross-population microbiome comparisons have shown that only a small set of

70 'core' gut bacteria are consistently detected across individuals within a population, and fewer

71 are shared across populations (Falony et al., 2016; Li et al., 2013; Qin et al., 2010; Tap et al., 
72 2009). Rather, the human gut microbiota is highly individualised, with each person harbouring

73 a characteristic microbial fingerprint which is distinct from that of others over time (Bergström

74 et al., 2014; De Muinck \& Trosvik, 2018; Faith et al., 2013; Turnbaugh et al., 2009). However,

75 given the unique ecology of industrialised humans, whether such patterns apply to non-human

76 wild vertebrates remains poorly understood. Cross-population studies in the wild examining to

77 what extent taxa are shared by conspecifics across geographical space, and at what scale this

78 might occur, are rare (Grieneisen et al., 2019; Lankau et al., 2012; Linnenbrink et al., 2013;

79 Smith et al., 2015; Funosas et al., 2021). Moreover, most wild animal microbiota studies are

80 cross-sectional or group-level, with few that have characterised the microbiota of repeat-

81 sampled individuals. Such longitudinal data are crucial for understanding microbiota stability at

82 both the individual and population level, and how this may be shaped by fluctuations in the

83 environment.

84 A growing number of studies have revealed strong seasonal dynamics in the gut microbiota of 85 wild animals (Kobayashi et al., 2006; Williams et al., 2013; Fogel, 2015; Springer et al., 2017;

86 Amato et al., 2014; Sun et al., 2016; Liu et al., 2019; Ren et al., 2017; Maurice et al., 2015;

87 Orkin et al., 2019). Pronounced seasonal microbiota dynamics have also been observed among

88 humans leading traditional, hunter-gatherer lifestyles (Davenport et al., 2014; Smits et al.,

89 2017). These findings contrast with those from industrialised humans, where microbiota

90 composition is considered relatively stable in adulthood (Faith et al., 2013). The causes of such

91 seasonal dynamics are not fully understood, but existing studies have implicated seasonal

92 shifts in diet (Amato et al., 2014a; Ren et al., 2017), or hibernation (Carey, Walters, \& Knight,

93 2013; Sommer et al., 2016). However, in many studies the fine-scale seasonal dynamics are not

94 well-understood as temporal resolution is relatively course (e.g. winter vs. summer), and the

95 extent to which seasonal dynamics are idiosyncratic or repeatable across years or populations

96 of a species remains unknown. Understanding the repeatability of such seasonal dynamics

97 across time and space is a key step towards understanding their potential functional

98 significance. If such temporal dynamics are general across populations and years, this would 
99 strengthen the support for a broadly applicable underlying process (for example a predictable

100 environmental or intrinsic host seasonal change), and allow for the possibility that such

101 changes could provide the host with repeatable adaptive seasonal plasticity (Alberdi et al.,

102 2016; Amato et al., 2014a).

103 Here, we present a longitudinal analysis of gut microbiota dynamics across two wild wood

104 mouse (Apodemus sylvaticus) populations, together with a comparison from a wild-derived

105 captive colony of the same host species. Wood mice are an ideal study system for questions

106 relating to the wild host microbiome, as they are a common species that are amenable to

107 identifiable capture-mark-recapture protocols. Furthermore, a previous study on a UK wood

108 mouse population has described seasonal shifts in gut microbiota composition, occurring

109 between summer and autumn (Maurice et al., 2015). We provide comprehensive analyses of

110 intra- and inter-individual host level variation and elucidate the factors associated with this

111 variation in each wild population.

113 Methods

114 Sample collection

115 Rodents were live-trapped using a capture-mark-recapture study design in two mixed

116 deciduous woodlands approximately $50 \mathrm{~km}$ apart in the UK: Wytham Woods, Oxfordshire

117 ('Wytham', $51^{\circ} 46^{\prime} \mathrm{N}, 1^{\circ} 20^{\prime} \mathrm{W}$ ) and Silwood Park, Berkshire ('Silwood', 51 $24^{\circ} \mathrm{N}, 0^{\circ} 38^{\prime} \mathrm{W}$ ).

118 Fieldwork methods were consistent across sites, with trapping performed for one night every

119 2-4 weeks across all seasons. In Silwood, trapping occurred on a single 2.47 ha grid over a one-

120 year period (Nov 2014 to Dec 2015), while in Wytham trapping took place over a three-year

121 period (Oct 2015 to Oct 2018), initially on a 1 ha grid that was then expanded to 2.4 ha in

122 October 2017. Wood mice (Apodemus sy/vaticus) were the most commonly caught rodent at

123 both sites, with yellow-necked mice (Apodemus flavicollis) and bank voles (Myodes glareolus) 
124 also captured. Small Sherman live traps were baited with six peanuts and a slice of apple, with

125 sterile non-absorbent cotton wool provided for insulation. Traps were set in alternate $10 \times 10 \mathrm{~m}$

126 grid cells at dusk and collected at dawn. All newly captured rodents were injected

127 subcutaneously with a PIT tag for identification purposes, with ear notches used as a back-up

128 form of identification. The following information was recorded for all captures: species, ID, sex,

129 age (juvenile, sub-adult or adult, according to size and pelage characteristics), reproductive

130 state (active or inactive according to signs of reproduction such as testes size in males,

131 perforation, pregnancy or lactation in females), body mass (to the nearest $0.1 \mathrm{~g}$ ), subcutaneous

132 fat score (0-4, measured by palpating the lower spine and hips). All animals were released

133 within the $10 \times 10 \mathrm{~m}$ grid cell of their capture. Faecal samples (approximately $40-300 \mathrm{mg}$ ) were

134 collected from the bedding material with sterilized tweezers, and frozen at $-80^{\circ} \mathrm{C}$ within 10

135 hours of trap collection for molecular work. Traps that showed any sign of animal contact

136 (both traps that held captured animals and those where an animal had interfered with but not

137 triggered it) were washed thoroughly with $20 \%$ bleach solution between trapping sessions to

138 prevent cross-contamination. Live-trapping work was conducted with institutional ethical

139 approval, and at Wytham under Home Office licence PPL-I4C48848E.

140 Faecal samples were also collected from an outbred, captive colony of wood mice kept at the

141 University of Edinburgh, and included for comparison to wild populations. This colony was

142 initiated over 30 years ago at the University of Oxford (Department of Zoology) using wild-

143 caught mice from the local area (presumed to be Wytham Woods, where department

144 members were trapping rodents at the time, though this has not been confirmed) and bred

145 there for several decades (Hughes et al., 2010). Captive wood mice included in this study were

146 sampled as part of a diet shift experiment carried out in 2017 under Home Office Project

147 license 70/8543. Further details about the colony and experiment are given in the Supporting

148 Information. 
150 Genomic DNA was extracted from faecal samples using Zymo Quick-DNA faecal/soil Microbe

15196 (96-well plate format) extraction kits, according to manufacturer instructions. A 254bp

152 region of the bacterial $16 \mathrm{~S}$ rRNA gene (V4 region) was amplified using primers 515F/806R

153 (Caporaso et al., 2011; Table S1). Library preparations followed a two-step (tailed-tag)

154 approach with dual-indexing ( $D^{\prime}$ Amore et al., 2016) and sequencing was performed on an

155 Illumina ${ }^{\circledR}$ MiSeq with $250 \mathrm{bp}$ paired-end reads, at the Centre for Genomic Research in

156 Liverpool. Samples were extracted in 17 batches and sequenced in 5 lanes, with some Wytham

157 and colony samples extracted and sequenced together, while samples from Silwood were

158 extracted and sequenced separately with minor adjustments to the protocol (Supporting

159 Information). To avoid further batch effects influencing results, wild samples from each

160 trapping session and colony samples from each timepoint and animal were evenly distributed

161 across extraction plates. Full details of $16 \mathrm{~S}$ rRNA sequencing methods are provided in

162 Supporting Information.

163 Bioinformatics

164 Raw sequence data were processed through the DADA2 pipeline (v1.6) in R to infer amplicon

165 sequence variants (ASVs; Callahan et al., 2016, 2017). This was done separately for each Miseq

166 run, but using identical parameters. In brief, reads were trimmed and filtered for quality, ASVs

167 inferred and putative chimeras removed before taxonomic assignment performed using the

168 v128 SILVA reference database. Full details of the bioinformatics pipeline are in Supporting

169 Information. A single phyloseq object (McMurdie \& Holmes, 2013) containing data from all

170 three populations was then created for further processing and analyses. ASVs taxonomically

171 assigned as chloroplast or mitochondria were removed, after which the dataset contained

172 11,404 ASVs. The R package 'iNEXT' (Chao et al., 2014; Hsieh et al., 2016) was used to examine

173 sample completeness and rarefaction curves, which showed that sample completeness

174 plateaued at approximately 8000 reads (Fig. S1). 31 samples with read counts below this

175 threshold were removed, leaving data from 1052 samples and 281 mice overall (Wytham: $n=$ 
176448 samples from 178 individuals; Silwood: $n=253$ from 75 individuals; captive colony: $n=351$

177 from 28 individuals), with a mean read count of 40,478 (range $8841-150,932$ ). For beta

178 diversity analyses, further taxon filtering was performed by retaining only those ASVs with

179 more than 1 copy in at least $1 \%$ of samples, to guard against the influence of potential PCR or

180 sequencing artefacts, or contaminants. After this additional ASV filtering step, the dataset

181 contained 2662 ASVs.

182 Statistical analyses

183 All statistical analyses were performed in R version 3.5.3 ( $R$ Core Team, 2019).

184 Defining core taxa

185 To delimit core taxa, we estimated each ASV's population-wide prevalence as the proportion of

186 samples in which it was detected, in a dataset containing one randomly selected sample per

187 individual, averaged over 100 iterations. We also estimated each ASV's within-individual

188 persistence, as the proportion of samples per repeat-sampled individual it was detected in, and

189 taking the mean across mice sampled at least three times ( $\mathrm{n}=57$ in Wytham, with mean $\mathrm{N}$

190 captures $4.86 \pm 1.94$ s.d., $n=39$ in Silwood with mean $N$ captures $=5.95 \pm 1.95$ s.d.).

191 Alpha-diversity analyses

192 ASV richness was estimated using the R package 'breakaway' (Willis \& Bunge, 2015), using a

193 dataset for which taxon filtering was limited to the removal of ASVs assigned as chloroplast or 194 mitochondria.

195 Beta-diversity analyses

196 ASV read counts were normalised to relative abundance per sample before calculating 197 pairwise dissimilarity indices among samples (Bray-Curtis dissimilarity; McKnight et al., 2019), 198 which was subsequently used in principal coordinates analysis (PCOA) and permutational 199 analysis of variance (PERMANOVA) per population. To assess the decay of within-individual 
200 community similarity over time (time-decay), we modelled the log-linear change in community

201 structure (pairwise Bray-Curtis dissimilarity) with the number of days between samples.

202 Community dissimilarities were converted to similarities (1- dissimilarity) and log-transformed.

203 The rate of change at different time lags was calculated by dividing the Bray-Curtis dissimilarity

204 by the time between sampling points to obtain an average rate of change for each time lag

205 (Faith et al., 2013; Shade et al., 2013). To compare inter- and intra-individual variation in gut

206 microbiota composition, pairwise dissimilarity values were compared across different types of

207 sample pair, using Wilcoxon tests with 1,000 permutations: those collected from the same

208 individual at different timepoints ('Same mouse') and those collected from different

209 individuals within the same trapping session ('Same date').

210 To analyse temporal change in microbiota composition, generalised additive mixed models

211 (GAMM) were run in package 'mgcv', using sample scores along the first axis of a Bray-Curtis

212 PCoA (PC1) as the response variable. A cyclic cubic spline was fitted for day of the year to

213 model within-year seasonal patterns, with corresponding sample-level host and

214 methodological terms included as covariates (age, sex, reproductive status, body mass, body

215 condition, read count). Year and MiSeq run were also fitted as covariates in analyses for

216 Wytham. Animal ID was included as a random factor to control for repeated measures. The

217 same model structure was used to assess seasonal patterns in microbial richness.

218 To test the relative effects of host, environmental and methodological factors on variation in

219 gut community structure, a marginal PERMANOVA was performed using the adonis2 function

220 in package 'vegan' (Oksanen et al., 2019) with 999 permutations, on a subset of data including

221 one randomly chosen sample per individual, to avoid pseudoreplication. Subsequent tests for

222 homogeneity of variance between groups of significant terms were performed using the

223 betadisper function. To circumvent the decrease in sample size from subsetting to one sample

224 per individual, we also performed a partial redundancy analysis (pRDA) on Hellinger-

225 transformed community data, using the same explanatory terms as used in the PERMANOVA, 
226 but with individual ID used as a condition. The marginal significance of the explanatory terms

227 were then calculated under the reduced model.

228 To identify bacterial taxa (ASVs) involved in detected trends, we used Random Forest models

229 with default parameters (package 'randomForest'). 'IncNodePurity' was used as a measure of

230 ASV importance.

\section{Results}

\section{Assessing core microbes across populations}

234 Across all three (wild and captive) populations, the gut microbiota of wood mice was broadly 235 comparable in terms of proportional abundances of high-level taxa, being dominated by the 236 orders Lactobacillales, Clostridiales and Bacteroidales (Fig. 1a). At the order level, Wytham 237 mice had a slightly higher relative abundance of Bacteroidales, while Silwood mice had a 238 slightly higher relative abundance of Lactobacillales. The extent to which a set of 'core' 239 microbes common to all populations was identifiable declined as higher levels of bacterial 240 taxonomic resolution were considered (Fig. 1b). Nine phyla were detected in total, eight of

241 which were detected in all populations; Spirochaetes was only found in Wytham and the 242 colony while Elusimicrobia was found only in Silwood. The majority (74\%) of identified 243 bacterial orders were common to all populations and similarly $62 \%$ of identified genera were 244 shared by all three populations, wild and lab (Fig. 1b). However, strong taxonomic divergence 245 was detected among populations at the ASV level; no ASVs were shared between the two wild 246 mouse populations, nor between Silwood wild mice and the captive colony (Fig. 1b). This

247 finding was unaltered when taxon filtering was minimal and limited to the removal of reads 248 assigned as chloroplast/mitochondria. In contrast, more than half the ASVs seen in wild mice 249 from Wytham were also detected in the captive colony $(52.1 \%$ of Wytham and $95 \%$ of Colony 250 ASVs; Fig. 1b). Differences in the proportion of taxa shared by populations were unaltered 
251 when these comparisons were made using samples sequenced in the same vs. different runs

252 (Fig. S2), indicating they are very unlikely to be an artifact of the fact Silwood samples were 253 sequenced separately. On average, a pair of mice from Wytham and the colony shared $8.4 \%$

254 ASVs (mean Jaccard Index), while the proportion of shared ASVs within a population was 255 slightly higher (Wytham=11.5\%, Colony=23.2\%, Silwood=18.0\%). The ASVs common to

256 Wytham and colony mice came from a broad range of bacterial families, representative of 257 those observed in each population (Fig. S3).

\section{Assessing core microbes within populations}

There were no ASVs shared by all samples within each population, i.e. no population-specific 'core microbiota'. To explore how we might define a set of 'core' microbiota members using a

261 less strict criterion than universal colonisation, we first examined the relationship between

262 ASV prevalence across hosts and persistence within them. In both wild populations, there was

263 a strong positive correlation between the prevalence and persistence of ASVs (Spearman's

264 rank correlation; Wytham; rho=0.932, $p<0.001$, Silwood; rho=0.946, $p<0.001$, Fig. S4). ASVs

265 that were both prevalent and persistent ( $>50 \%$ for each) formed a taxonomically biased

266 subset, being enriched for the order Bacteroidales (and slightly for Lactobacillales) compared

267 to the total set of ASVs in each wild population (Fig. S4).

268 Defining core taxa using both prevalence and abundance thresholds (present in at least $60 \%$ 269 samples at $0.1 \%$ relative abundance or more) identified a similar set of ASVs in each 270 population. In Wytham, this gave a set of 23 core ASVs, belonging to Bacteriodales S24-7 group 271 ( $n=16,69 \%)$, Peptococcaceae $(n=1)$, Ruminococcaeae $(n=3)$ and Lactobacillaceae $(n=3) . \quad$ Only 272 two of these 'core' ASVs could be identified to genus level and belonged to Lactobacillus and 273 Ruminiclostridium 9, respectively. Using the same core definition, the Silwood population 274 contained 21 core ASVs, belonging largely to the Muribaculaceae $(n=11)$ as well as 275 Ruminococcaceae $(n=4), \quad$ Lactobacillaceae $(n=4), \quad$ Helicobacteraceae $(n=1) \quad$ and 276 Coriobacteraceae $(n=1)$. 


\section{Predictors of gut microbiota composition}

278 In both wild populations, month was the strongest predictor of gut microbiota composition, 279 explaining approximately twice as much variation as all host factors combined in marginal 280 PERMANOVAs (Table 1). Although population age structure varies seasonally (Fig. S5), the 281 seasonal effect detected here is independent of this, as host age was controlled for in the 282 model (Table 1). Microbiota composition also differed between years in Wytham. Weaker 283 effects of host factors (body mass and reproductive status) were detected, but each in only 284 one of the two populations (Table 1). Dispersion tests indicated that the effect of year may 285 have been influenced by dispersion differences in Wytham while the effect of month may also 286 contain some influence of dispersion in Silwood (Table 1). Results from the pRDA on Bray-

287 Curtis dissimilarity agreed with those from PERMANOVAs, in that month and year strongly 288 predicted gut microbiota variation in both wild populations, while measured host factors did 289 not (Table S3). However, the pRDA also revealed that individual ID (as a condition) explained 290 around half the total variation in each dataset ( $55.2 \%$ in Wytham and $48.1 \%$ in Silwood).

291 Repeatable seasonal restructuring of the microbiota

292 The major axis of microbiota compositional variation (PC1 from a Bray-Curtis PCoA, explaining $29312.89 \%$ of variation in Wytham and $20 \%$ in Silwood) shifted strongly between July, October 294 and February, and this seasonal pattern was remarkably consistent across both mouse 295 populations (Fig. 2a, c; Table S2). PC1 was not significantly predicted by any other host or 296 methodological fixed effects (Table S2). In both populations, seasonal changes in PC1 within 297 repeat-sampled individuals typically tracked population-level seasonal shifts, with few 298 exceptions (Fig. 2b, d; paired t-tests for Wytham Jun-Aug vs. Sept-Nov; $t=-5.152, p<0.001$, $299 \mathrm{n}=14$, Sept-Nov vs. Jan-March; $\mathrm{t}=2.676, \mathrm{p}=0.015, \mathrm{n}=20$, for Silwood Jun-Aug vs. Sept-Nov; $\mathrm{t}=-$ $3003.23, p=0.006, n=16$, Sept-Nov vs. Jan-March; $t=0.250, p=0.805, n=26)$. Of the few individuals 301 that were not consistent with the population-level patterns, there were no unique or unusual 
302 observations in the measured host traits, though repeat captures were too few between these

303 months to formally test factors associated with individual differences in the direction or

304 magnitude of seasonal change in PC1. The pattern of seasonal change in microbiota

305 composition was similar when using the PC1 of Jaccard-based PCoA (a presence/absence-

306 based distance metric) as the response, although in Silwood the Jaccard-based PC1 explained a

307 lower proportion of gut community variation than Bray-Curtis PC1 (Fig. S6). This shows that gut

308 microbiota seasonality primarily consists of changes in relative abundance rather than

309 turnover, as can be seen in Sankey plots showing the flux of ASVs between seasons (Fig. S7).

310 Despite this prominent seasonal shift in microbiota composition, the relative abundances of

311 bacterial families did not change dramatically across the year (Fig. S8). Exploratory analyses

312 also showed that while in Silwood the second and third PCoA axes showed some temporal

313 fluctuations during the same periods that PC1 shifted (oscillation between July and December),

314 in Wytham other PCoA axes besides PC1 did not show strong seasonal variation (Fig. S9).

315 Seasonal changes in mean gut microbiota richness were weaker compared to seasonal changes

316 in composition. In models where richness was the response, day of the year was non-

317 significant for Wytham mice, and in Silwood was significant though explained a relatively small

318 amount of variation compared to similar analyses of composition (Fig. S10, Wytham:

319 approximate significance of smoothed date term $F=0.00$, edf $<0.001, p=0.516$, adjusted

$320 \mathrm{R}^{2}=0.09, \mathrm{n}=328$; Silwood: approximate significance of smoothed date term; $\mathrm{F}=1.767$,

321 edf=4.722, $p<0.001$, adjusted $\left.R^{2}=0.14, n=185\right)$.

322 We used random forest regressions to explore which bacterial taxa might drive the consistent

323 seasonal changes in PC1 seen in each wild population. For both populations, the top 6 ASVs

324 predicting PC1 values belonged to three bacterial families: Lactobacillaceae, Muribaculaceae

325 and Ruminococcaceae, with the exact order of importance for these families differing slightly

326 between populations (Fig. 3). In Wytham, the model explained $92.11 \%$ of variation in PC1

327 values, and the most important ASVs predicting PC1 belonged to Ruminococcaceae, followed 
328 by Muribaculaceae, Lactobacillaceae and Bifidobacteriaceae (Fig. 3). In Silwood, the model

329 explained $93.31 \%$ in PC1 and all top four ASVs in predicting PC1 belonged to Lactobacillaceae,

330 followed by ASVs belonging to Muribaculaceae and Ruminococcaceae (Fig. 3). The bacterial

331 ASVs most strongly associated with variation along Silwood PC2 and PC3 belonged to

332 Lactobacillaceae, Ruminococcaceae, Lachnospiraceae and Muribaculaceae (Fig S11).

333

334 Individuality and seasonal convergence in the gut microbiota

335 Despite strong and repeatable seasonal shifts in population-level gut microbiota composition

336 (Fig. 2), we nonetheless detected a large degree of individuality in the microbiota of wild mice.

337 In each wild population, individuals were, on average, more similar in gut microbiota

338 composition (Bray-Curtis dissimilarity) to themselves at other time-points than to other mice

339 sampled on the same day (Fig. 4a; permutation tests; Wytham observed U-statistic $=4222156$,

$340 \mathrm{p}<0.001$; Silwood observed U-statistic $=1802548, \mathrm{p}<0.001$ ). This signal of individuality decayed

341 with increasing sampling interval (Fig. 4b; log-linear model for Wytham; $F=107.6_{(1,1268)}$,

$342 p<0.001$, adjusted $R^{2}=0.078$, Silwood; $F=17.51_{(1,918)}, p<0.001$, adjusted $\left.R^{2}=0.018\right)$, with the rate

343 of decay highest at short time intervals and less strong as the sampling interval increased

344 (Table S4).

345 Although there was a strong signal of individuality overall (intra-individual variation is on

346 average lower than inter-individual variation), this does not account for temporal dynamics in

347 the community composition. We therefore also assessed seasonal changes in inter-individual

348 variation (Bray-Curtis dissimilarity) to determine whether the signal of individuality remains

349 strong throughout the year. In general, there tended to be more convergent gut microbiotas

350 between individuals in winter but more inter-individual variation in the summer months (Fig.

$3514 \mathrm{c}$ ). This inter-individual variation remained higher than the average within-individual variation

352 for most of the year (Fig. 4c), showing that the signal of individuality remains consistently

353 strong. However, in Wytham, the seasonal convergence of gut microbiota composition seen in 
354 late winter/ early spring was sufficiently strong to override the signal of individuality (Fig. 4c);

355 mice sampled in February were more similar to each other than they were to themselves at

356 other times of the year (permutation test; observed U-statistic $=223084, p<0.001$ ). A weaker

357 convergence was observed in the Silwood population at this same time of year (late winter/

358 early spring), which nullified the signal of individuality i.e. mice caught at this time were no

359 more similar to themselves across the year than they were to others sampled simultaneously

360 (permutation test; observed U-statistic $=33916, p=0.712$ ). This reduction in inter-individual

361 variation was observed again in October in Silwood (permutation test; observed U-statistic=

$36230240, p=0.046$ ) but not in Wytham (Fig. 4c).

\section{Discussion}

364 Here we have provided an in-depth examination of natural variation in the gut microbiota of a

365 wild mammalian host, the wood mouse, using detailed longitudinal analyses from multiple

366 populations and years. We found no universal core microbial community (shared by all

367 members of the species) when considering bacterial ASVs, genera or families across all

368 populations. The proportion of bacterial taxa shared between host populations decreased with

369 increasing taxonomic resolution, and at the finest resolution of ASVs there was a high degree

370 of population-specificity in the taxa identified, suggesting a high amount of turnover in the

371 species/strains present even between populations $50 \mathrm{~km}$ apart. Geographic variation in gut

372 microbial communities has been observed in a number of host species, with processes such as

373 differences in diet and/or host genetics (Goertz et al., 2019; Smith et al., 2015; Suzuki et al.,

374 2019), environmental differences such as soil properties (Grieneisen et al., 2019) or ecological

375 drift (Lankau et al., 2012; Linnenbrink et al., 2013; Stothart et al., 2020) cited as drivers of this

376 variation. In contrast, the captive colony of wood mice retained approximately half of the

377 diversity of the wild population from which it likely originated, indicating a slow rate of taxon

378 loss over time in captivity with minimal input of new microbes as has been observed in other

379 mouse systems (Kohl \& Dearing, 2014; Sonnenburg et al., 2016). The patterns observed here 
380 were not simply an artefact of the sequencing methodology, as the subsets of Wytham and

381 colony samples sequenced in the same or different runs shared similar proportions of taxa in

382 common. The degree of shared ASVs between the wild and captive populations studied here

383 could be driven either by the degree of host or microbial genetic divergence between

384 populations (highest between the two wild populations, but lower between the colony and

385 Wytham), or by variability of environmental microbial input, which is high between wild

386 populations but limited in captivity.

387 Associations between microbiota structure and host body mass (Sommer et al., 2016;

388 Turnbaugh et al., 2006), age (Bennett et al., 2016; Degnan et al., 2012) and reproductive status

389 (Amato et al., 2014b) have been detected previously. However, here we show that sampling

390 month remains a strong predictor of microbiota variation after accounting for these intrinsic

391 host factors, and seasonality is therefore likely driven by external factors in this system. These

392 seasonal microbiota changes were much stronger than any host-associated effects examined,

393 with sampling month explaining more than twice the variation in community structure than

394 any other variable tested, and more than all host-related variables combined. This suggests

395 that associations with host factors are relatively weak compared to external drivers of gut

396 microbiota variation, in line with studies on other wild animal populations (Goertz et al., 2019;

397 Ren et al., 2017) and humans (Rothschild et al., 2018).

398 By sampling with high temporal resolution throughout multiple years, we detected a strong 399 seasonal oscillation in microbiota structure that consistently occurred between July and

400 February. This seasonal shift occurred independently of host age, sex or reproductive status,

401 and was also observed within individuals. The timing and form of this seasonal microbiota 402 restructuring was remarkably consistent across both populations and all years examined, and 403 strongly resembled patterns detected in a two-year study of a separate UK wood mouse 404 population (Maurice et al., 2015). Other studies in wild animals have similarly reported a 405 dominant role of season in restructuring the gut microbiota compared to host factors 406 (Kobayashi et al., 2006; Williams et al., 2013; Fogel, 2015; Springer et al., 2017; Amato et al., 
407 2014a; Sun et al., 2016; Liu et al., 2019; Ren et al., 2017; Maurice et al., 2015). However, this is

408 the first time such seasonality has been shown to have such a consistent pattern across

409 multiple populations and years. The striking repeatability of this seasonal pattern across

410 populations suggests common drivers are at play. These could include predictable seasonal

411 shifts in diet, parasitic infection, host physiology or social behaviour (David et al., 2014;

412 Perofsky et al., 2017; Kreisinger et al., 2015). Hibernation has been linked to restructuring of

413 the gut microbiota in ground squirrels and brown bears (Carey et al., 2013; Sommer et al.,

414 2016). Although wood mice do not hibernate, they can enter short periods of torpor during

415 colder months, with decreased body temperature and metabolic activity. However, this

416 explanation of seasonal restructuring in the wood mouse gut microbiota is unlikely, as the

417 major seasonal restructuring begins in summer. Therefore, seasonal shifts in diet are a strong

418 candidate mechanism. Wood mice are omnivorous species that in woodland habitats rely on

419 nuts which mature in summer and are cached for consumption until the following spring, such

420 that their diet shows a strong increase in this material during the autumn/winter (Watts 1968).

421 This predictable influx of nuts to the diet could be what drives consistent seasonal

422 restructuring across populations and years in woodland habitats, a hypothesis that could be

423 tested in this system through direct measurement of seasonal diet-microbiota links in future.

424 Despite strong seasonal changes in the gut microbiota that were observed consistently across

425 individuals and populations, a high degree of individuality in the gut microbiota was still

426 detectable. On average, individuals were more similar to themselves over time than to other

427 individuals sampled simultaneously. Such individuality could be explained by genetic

428 differences (Benson et al., 2010), or persistent environmental or behavioural differences, for

429 example in habitat, dietary preferences or parasite burden. This individuality signal weakened

430 as sampling interval increased, suggesting that temporal changes (e.g. environmental or aging-

431 related) can affect the strength of this individual gut microbial signature (Faith et al., 2013).

432 We also found seasonal variation in the degree of microbiota similarity between hosts, with

433 the gut microbiota being more similar among mice caught in winter and early spring. This 
434 convergence of gut microbiotas in February-March was sufficiently strong to reduce the signal

435 of individuality and even temporarily override it within the Wytham population. This could be

436 due to a more homogenous diet at this time of year, when wood mice are thought to eat

437 mainly cached seeds and nuts, compared to later spring/summer, when a more diverse array

438 of both plant and animal items is available (Watts 1968). Alternatively, seasonal convergence

439 could be driven by population density and/or social interactions, which vary seasonally and

440 could influence rates of microbial transmission between hosts (Raulo et al., 2021; Wolton,

441 1985). Seasonal convergence between individual hosts in relation to the signal of individuality

442 in the gut microbiota has not been previously examined. However, this could have

443 consequences for the host population in terms of how well the gut microbiota can provide

444 resilience to perturbations such as altered food availability or pathogen invasion at different

445 times of the year.

446 The repeatability of seasonal microbiota dynamics across populations observed here is 447 particularly striking given these two host populations possessed no bacterial ASVs in common.

448 Some, but not all, of the higher order taxa associated with seasonal changes were consistent

449 across populations. For instance, Lactobacillaceae ASVs were implicated in both Wytham and

450 Silwood, consistent with previous work (Maurice et al., 2015), while members of

451 Ruminococcaceae, Bacteroidales S24-7 group, Bifidobacteriaceae and Lachnospiraceae were

452 important in Wytham only. Thus across populations, different bacterial taxa appear to respond

453 synchronously to the same seasonal change. This suggests there may be a level of functional

454 redundancy in the gut microbial taxa of wood mice that respond seasonally at broad

455 geographical scales. Further functional studies, for example using metagenomic or

456 metatranscriptomic approaches, would be valuable to illuminate what seasonal functions

457 these microbes might perform for the host, and the potential significance of such microbiome

458 shifts in providing hosts living in variable environments with adaptive seasonal plasticity.

\section{Acknowledgements}


460 This work was funded by a NERC fellowship (NE.L011867/1) to SCLK and a Royal Veterinary

461 College (RVC) studentship to KJM (supervised by SCLK and JPW). Our thanks go to Tim Coulson

462 for enabling the fieldwork at Wytham Woods, and to Amy Pedersen for enabling the sample

463 collection in the captive wood mouse colony.

464 Conflicts of Interest

465 The authors declare no conflicts of interest.

\section{Author Contributions}

467 This work was supported by a NERC fellowship (NE.L011867/1) to SCLK and funding from the

468 European Research Council (ERC) under the European Union's Horizon 2020 research and 469 innovation programme (grant agreement $n^{\circ} 851550$ ). KJM was supported by a Royal 470 Veterinary College (RVC) studentship (supervised by SCLK and JPW). Our thanks go to Tim

471 Coulson for enabling the fieldwork at Wytham Woods, and to Amy Pedersen for enabling

472 sample collection in the captive wood mouse colony.

\section{Data Availability Statement}

474 All relevant data will be made available on a suitable repository.

\section{References}

476 Alberdi, A., Aizpurua, O., Bohmann, K., Zepeda-Mendoza, M. L., \& Gilbert, M. T. P. (2016). Do Vertebrate Gut Metagenomes Confer Rapid Ecological Adaptation? Trends in Ecology and Evolution, 31(9), 689-699. https://doi.org/10.1016/j.tree.2016.06.008

Amato, K. R., Leigh, S. R., Kent, A., Mackie, R. I., Yeoman, C. J., Stumpf, R. M., ... Garber, P. A. 480 (2014a). The Gut Microbiota Appears to Compensate for Seasonal Diet Variation in the 481 Wild Black Howler Monkey (Alouatta pigra). Microbial Ecology, 69, 434-443. https://doi.org/10.1007/s00248-014-0554-7 
483 Amato, K. R., Leigh, S. R., Kent, A., Mackie, R. I., Yeoman, C. J., Stumpf, R. M., ... Garber, P. A.

484 (2014b). The role of gut microbes in satisfying the nutritional demands of adult and

485 juvenile wild, black howler monkeys (Alouatta pigra). American Journal of Physical

486 Anthropology, 155(4), 652-664. https://doi.org/10.1002/ajpa.22621

Bennett, G., Malone, M., Sauther, M. L., Cuozzo, F. P., White, B., Nelson, K. E., ... Amato, K. R. (2016). Host age, social group, and habitat type influence the gut microbiota of wild ringtailed lemurs (Lemur catta). American Journal of Primatology, 78(8), 883-892. https://doi.org/10.1002/ajp.22555

Benson, A. K., Kelly, S. A., Legge, R., Ma, F., Low, S. J., Kim, J., ... Pomp, D. (2010). Individuality in gut microbiota composition is a complex polygenic trait shaped by multiple environmental and host genetic factors. Proceedings of the National Academy of Sciences

Bergström, A., Skov, T. H., Bahl, M. I., Roager, H. M., Christensen, L. B., Ejlerskov, K. T., ... Licht, of the United States of America, 107(44), 18933-18938. https://doi.org/10.1073/pnas.1007028107 intestinal pathogens. Nat Rev Immunol, 13(11), 790-801. https://doi.org/10.1038/nri3535 operational taxonomic units in marker-gene data analysis. The ISME JournaI, 11(12), 2639-2643. https://doi.org/10.1038/ismej.2017.119 
509 Caporaso, J. G., Lauber, C. L., Costello, E. K., Berg-Lyons, D., Gonzalez, A., Stombaugh, J., ... Knight, R. (2011). Moving pictures of the human microbiome. Genome Biology, 12(5), R50. https://doi.org/10.1186/gb-2011-12-5-r50

512 Carey, H. V, Walters, W. A., \& Knight, R. (2013). Seasonal restructuring of the ground squirrel gut microbiota over the annual hibernation cycle. Am J Physiol Regul Integr Comp Physiol,

Chao, A., Colwell, R. K., Gotelli, N. J., Hsieh, T. C., Sander, E. L., Ma, K. H., ... Ellison, A. M.

D’Amore, R., ljaz, U. Z., Schirmer, M., Kenny, J. G., Gregory, R., Darby, A. C., ... Hall, N. (2016). A

Davenport, E. R., Mizrahi-Man, O., Michelini, K., Barreiro, L. B., Ober, C., \& Gilad, Y. (2014). comprehensive benchmarking study of protocols and sequencing platforms for $16 \mathrm{~S}$ rRNA community profiling. BMC Genomics, 17(1), 0-20. https://doi.org/10.1186/s12864-0152194-9

De Muinck, E. J., \& Trosvik, P. (2018). Individuality and convergence of the infant gut microbiota during the first year of life. Nature Communications, 9, 2233. https://doi.org/10.1038/s41467-018-04641-7 
533 Degnan, P. H., Pusey, A. E., Lonsdorf, E. V, Goodall, J., Wroblewski, E. E., Wilson, M. L., ...

534 Ochman, H. (2012). Factors associated with the diversification of the gut microbial

535 communities within chimpanzees from Gombe National Park. Proceedings of the National

536 Academy of Sciences of the United States of America, 109(32), 13034-13039.

537 https://doi.org/10.1073/pnas.1110994109

538 Faith, J. J., Guruge, J. L., Charbonneau, M., Subramanian, S., Seedorf, H., Goodman, A. L., ...

539 Gordon, J. I. (2013). The long-term stability of the human gut microbiota. Science,

$540 \quad 341(6141), 1237439$. https://doi.org/10.1126/science.1237439

541 Falony, A. G., Joossens, M., Wang, J., \& Darzi, Y. (2016). Population - level analysis of gut

542 microbiome variation. Science, 352(6285), 560-564.

543 Flint, H. J., Scott, K. P., Louis, P., \& Duncan, S. H. (2012). The role of the gut microbiota in

544 nutrition and health. Nature Reviews Gastroenterology \& Hepatology, 9(10), 577-589.

545 https://doi.org/10.1038/nrgastro.2012.156

546 Fogel, A. T. (2015). The gut microbiome of wild lemurs: A comparison of sympatric lemur catta

547 and propithecus verreauxi. Folia Primatologica, 86, 85-95.

$548 \quad$ https://doi.org/10.1159/000369971

549 Funosas, G., Margarit, X. T., Castro, F., Villafuerte, R., Mateos, M. D., Rouco, C., \& Casamayor,

550 E. O. (2021). Individual fate and gut microbiome composition in the European wild rabbit

551 (Oryctolagus cuniculus ). Scientific Reports, 11, 766. https://doi.org/10.1038/s41598-020-

$552 \quad 80782-4$

553 Goertz, S., Menezes, A. B. De, Birtles, R. J., Id, J. F., Lowe, E., Maccoll, A. D. C., ... Taylor, C. H.

554 (2019). Geographical location influences the composition of the gut microbiota in wild

555 house mice ( Mus musculus domesticus ) at a fine spatial scale. PLOS ONE, 14(9),

$556 \quad$ e0222501.

557 Grieneisen, L. E., Charpentier, M. J. E., Alberts, S. C., Blekhman, R., Bradburd, G., Tung, J., ... 
Archie, E. A. (2019). Genes, geology and germs[?]: gut microbiota across a primate hybrid zone are explained by site soil properties, not host species. Proceedings of the Royal Society B, 286, 20190431.

Hsieh, T. C., Ma, K. H., \& Chao, A. (2016). iNEXT: an R package for rarefaction and extrapolation of species diversity (Hill numbers). Methods in Ecology and Evolution, 7(12), 1451-1456. https://doi.org/10.1111/2041-210X.12613

Hughes, D. J., Kipar, A., Milligan, S. G., Cunningham, C., Sanders, M., Quail, M. A., ... Stewart, J. P. (2010). Characterization of a novel wood mouse virus related to murid herpesvirus 4. Journal of General Virology, 91(4), 867-879. https://doi.org/10.1099/vir.0.017327-0

Kobayashi, Y., Koike, S., Miyaji, M., Hata, H., \& Tanaka, K. (2006). Hindgut microbes, fermentation and their seasonal variations in Hokkaido native horses compared to light horses. Ecological Research, 21(2), 285-291. https://doi.org/10.1007/s11284-005-0118-x

Kohl, K. D., \& Dearing, M. D. (2014). Wild-caught rodents retain a majority of their natural gut microbiota upon entrance into captivity. Environmental Microbiology Reports, 6(2), 191195. https://doi.org/10.1111/1758-2229.12118

Kreisinger, J., Bastien, G., Hauffe, H. C., Marchesi, J., \& Perkins, S. E. (2015). Interactions between multiple helminths and the gut microbiota in wild rodents. Philosophical Transactions of the Royal Society B: Biological Sciences, 370, 20140295. https://doi.org/10.1098/rstb.2014.0295

Lankau, E. W., Hong, P. Y., \& MacKie, R. I. (2012). Ecological drift and local exposures drive enteric bacterial community differences within species of Galápagos iguanas. Molecular Ecology, 21, 1779-1788. https://doi.org/10.1111/j.1365-294X.2012.05502.x

Li, K., Bihan, M., \& Methé, B. A. (2013). Analyses of the Stability and Core Taxonomic Memberships of the Human Microbiome. PLOS ONE, 8(5), e63139. https://doi.org/10.1371/journal.pone.0063139 
583 Linnenbrink, M., Wang, J., Hardouin, E. A., KUNZEL, S., Metzler, D., \& Baines, J. F. (2013). The

584 role of biogeography in shaping diversity of the intestinal microbiota in house mice.

585 Molecular Ecology, 22, 1904-1916. https://doi.org/10.1111/mec.12206

586 Liu, P. Y., Cheng, A. C., Huang, S. W., Chang, H. W., Oshida, T., \& Yu, H. T. (2019). Variations in 587 Gut Microbiota of Siberian Flying Squirrels Correspond to Seasonal Phenological Changes in Their Hokkaido Subarctic Forest Ecosystem. Microbial Ecology, 78(1), 223-231. https://doi.org/10.1007/s00248-018-1278-x

590

591

Maurice, C. F., Cl Knowles, S., Ladau, J., Pollard, K. S., Fenton, A., Pedersen, A. B., \& Turnbaugh, P. J. (2015). Marked seasonal variation in the wild mouse gut microbiota. The ISME Journal, 9, 2423-2434. https://doi.org/10.1038/ismej.2015.53

McKnight, D. T., Schwarzkopf, L., Zenger, K. R., Alford, R. A., Huerlimann, R., \& Bower, D. S. (2019). Methods for normalizing microbiome data: an ecological perspective. Methods in Ecology and Evolution, 10, 389-400. https://doi.org/10.1111/2041-210x.13115

McMurdie, P. J., \& Holmes, S. (2013). phyloseq: an R package for reproducible interactive analysis and graphics of microbiome census data. PloS One, 8(4), e61217. https://doi.org/10.1371/journal.pone.0061217

Oksanen, J., Blanchet, F. Guillaume Friendly, M., Kindt, R., Legendre, P., McGlinn, D., Minchin, P. R., ... Wagner, H. (2019). vegan: Community Ecology Package. Retrieved from https://cran.r-project.org/package=vegan

Orkin, J. D., Campos, F. A., Guadamuz, A., Melin, A. D., Myers, M. S., \& Hernandez, S. E. C. (2019). Seasonality of the gut microbiota of free-ranging white-faced capuchins in a tropical dry forest. The ISME Journal, 13, 183-196. https://doi.org/10.1038/s41396-0180256-0

Perofsky, A. C., Lewis, R. J., Abondano, L. A., Di Fiore, A., \& Meyers, L. A. (2017). Hierarchical social networks shape gut microbial composition in wild Verreaux's sifaka. Proceedings of 
the Royal Society B: Biological Sciences, 284(1868), 20172274.

https://doi.org/10.1098/rspb.2017.2274

Qin, J., Li, R., Raes, J., Arumugam, M., Burgdorf, K. S., Manichanh, C., ... Dore, J. (2010). A human gut microbial gene catalogue established by metagenomic sequencing. Nature, 464(4), 59-65. https://doi.org/10.1038/nature08821

Raulo, A., Allen, B. E., Troitsky, T., Husby, A., Firth, J. A., Coulson, T., \& Knowles, S. C. L. (2021). Social networks strongly predict the gut microbiota of wild mice. The ISME Journal, 15, 2601-2613. https://doi.org/10.1038/s41396-021-00949-3

Ren, T., Boutin, S., Humphries, M. M., Dantzer, B., Gorrell, J. C., Coltman, D. W., ... Wu, M. (2017). Seasonal, spatial, and maternal effects on gut microbiome in wild red squirrels. Microbiome, 5(1), 163. https://doi.org/10.1186/s40168-017-0382-3

Rothschild, D., Weissbrod, O., Barkan, E., Kurilshikov, A., Korem, T., Zeevi, D., ... Wijmenga, C. (2018). Environment dominates over host genetics in shaping human gut microbiota. Nature, 555, 7695. https://doi.org/10.1038/nature25973

Round, J. L., \& Mazmanian, S. K. (2009). The gut microbiota shapes intestinal immune responses during health and disease. Nat Rev Immunol, 9(5), 313-323. https://doi.org/10.1038/nri2515\nnri2515 [pii]

Shade, A., Caporaso, J. G., Handelsman, J., Knight, R., \& Fierer, N. (2013). A meta-analysis of changes in bacterial and archaeal communities with time. Ism, 7, 1493-1506. https://doi.org/10.1038/ismej.2013.54

Smith, C. C., Snowberg, L. K., Caporaso, J. G., Knight, R., \& Bolnick, D. I. (2015). Dietary input of microbes and host genetic variation shape among-population differences in stickleback gut microbiota. The ISME Journal, 9, 2515-2526. https://doi.org/10.1038/ismej.2015.64

Smits, S. A., Leach, J., Sonnenburg, E. D., Gonzalez, C. G., Lichtman, J. S., Reid, G., ... Sonnenburg, J. L. (2017). Seasonal Cycling in the Gut Microbiome of the Hadza Hunter- 
634 Sommer, F., Ståhlman, M., Ilkayeva, O., Arnemo, J. M., Kindberg, J., Josefsson, J., ... Bäckhed, F.

635 (2016). The Gut Microbiota Modulates Energy Metabolism in the Hibernating Brown Bear

$636 \quad$ Ursus arctos. Cell Reports, 14(7), 1655-1661.

637 https://doi.org/10.1016/j.celrep.2016.01.026 Sonnenburg, J. L. (2016). Diet-induced extinctions in the gut microbiota compound over generations. Nature, 529(7585), 212-215. https://doi.org/10.1038/nature16504

Stothart, M. R., Greuel, R. J., Gavriliuc, S., Henry, A., Wilson, A. J., Mcloughlin, P. D., \& Poissant, variation between winter and spring gut microbiota in free- ranging Tibetan Macaques (Macaca thibetana). Scientific Reports, 6, 26035. https://doi.org/10.1038/srep26035

Suzuki, T. A., Phifer-rixey, M., Mack, K. L., Sheeman, M. J., Lin, D., Ke, B., \& Nachman, M. W.

Tap, J., Mondot, S., Levenez, F., Pelletier, E., Caron, C., Furet, J., ... Leclerc, M. (2009). Towards 
658 Turnbaugh, P. J., Ley, R. E., Mahowald, M. a, Magrini, V., Mardis, E. R., \& Gordon, J. I. (2006).

659 An obesity-associated gut microbiome with increased capacity for energy harvest.

Nature, 444(7122), 1027-1031. https://doi.org/10.1038/nature05414

Williams, C. L., Willard, S., Kouba, A., Sparks, D., Holmes, W., Falcone, J., ... Brown, A. (2013). melanoleuca). Journal of Animal Physiology and Animal Nutrition, 97, 577-585. https://doi.org/10.1111/j.1439-0396.2012.01299.x 1049. https://doi.org/10.1111/biom.12332

Wolton, R. J. (1985). The ranging and nesting behaviour of Wood mice, Apodemus sylvaticus (Rodentia: Muridae), as revealed by radio-tracking. Journal of Zoology, 206(2), 203-222.

\section{Figures and Tables}

a)

673 b)

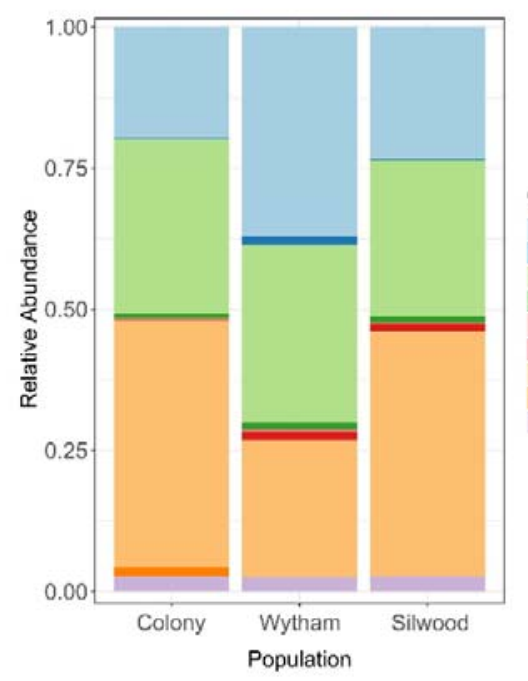

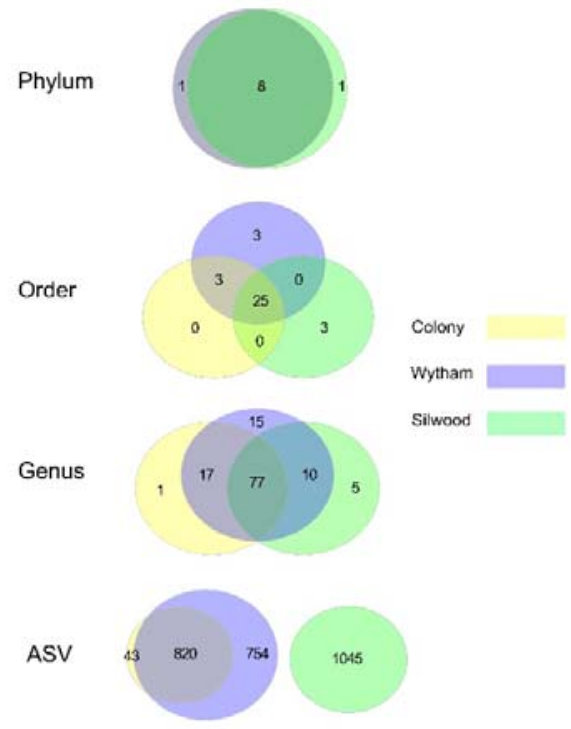


674 Figure 1. Gut microbiota composition and taxon sharing across populations of wood mice. a)

675 Comparison of microbiota composition at the Order level in samples from a captive colony

676 ( $n=351)$ and two wild populations, Wytham ( $n=448)$ and Silwood $(n=253)$. b) Euler diagrams

677 showing the number of shared and unique taxa across populations at four taxonomic levels:

678 Phylum, Order, Genus and ASV (amplicon sequence variant). Circle size is proportional to the

679 total number of taxonomic units, and circles are coloured by population: Colony (yellow),

680 Wytham (blue), Silwood (green).

681 Table 1. Predictors of microbiota composition in two populations of wild mice. Results are

682 shown from a marginal PERMANOVA on Bray-Curtis dissimilarity values. One randomly

683 selected sample per individual was included in the model (Wytham; $n=128$ and Silwood; $n=75$ ).

$684 \mathrm{p}$-values <0.05 are in bold. For significant terms (factors only), tests for multivariate

685 homogeneity of group dispersions were carried out and the results presented alongside the

686 PERMANOVA. The interaction between sex and reproductive status was fitted in a separate

687 model including this interaction term, results for all other terms are from a model without this

688 interaction.

\begin{tabular}{|c|c|c|c|c|c|c|c|c|}
\hline \multirow[b]{2}{*}{ Variable } & \multicolumn{4}{|c|}{ Wytham } & \multicolumn{4}{|c|}{ Silwood } \\
\hline & $d f$ & $\mathrm{~F}$ & $p$ & Partial $R^{2}$ & $d f$ & $\mathrm{~F}$ & $p$ & Partial $\mathrm{R}^{2}$ \\
\hline Read count & 1 & 1.338 & 0.104 & 0.009 & 1 & 0.629 & 0.895 & 0.007 \\
\hline MiSeq run & 3 & 1.460 & 0.006 & 0.030 & & & & \\
\hline Month & 11 & 1.758 & 0.001 & 0.133 & 10 & 1.584 & $0.001 \ddagger$ & 0.187 \\
\hline Year & 3 & 2.591 & $0.001+$ & 0.053 & & & & \\
\hline $\begin{array}{l}\text { Sex: Reproductive } \\
\text { status }\end{array}$ & 1 & 0.840 & 0.699 & 0.006 & 1 & 0.835 & 0.628 & 0.009 \\
\hline Sex & 1 & 0.908 & 0.598 & 0.006 & 1 & 0.786 & 0.684 & 0.009 \\
\hline $\begin{array}{l}\text { Reproductive } \\
\text { status }\end{array}$ & 1 & 1.320 & 0.126 & 0.009 & 1 & 1.731 & 0.066 & 0.020 \\
\hline Age & 2 & 1.001 & 0.428 & 0.014 & 2 & 0.930 & 0.563 & 0.022 \\
\hline Body mass & 1 & 1.382 & 0.065 & 0.010 & 1 & 1.018 & 0.382 & 0.012 \\
\hline Body condition & 4 & 0.944 & 0.642 & 0.026 & 4 & 0.928 & 0.615 & 0.044 \\
\hline
\end{tabular}

$689+$ and $\ddagger$ indicate terms for which dispersion tests indicated significant differences in dispersion among groups, with

$690 p=0.003$ and $p=0.01$ respectively

691 


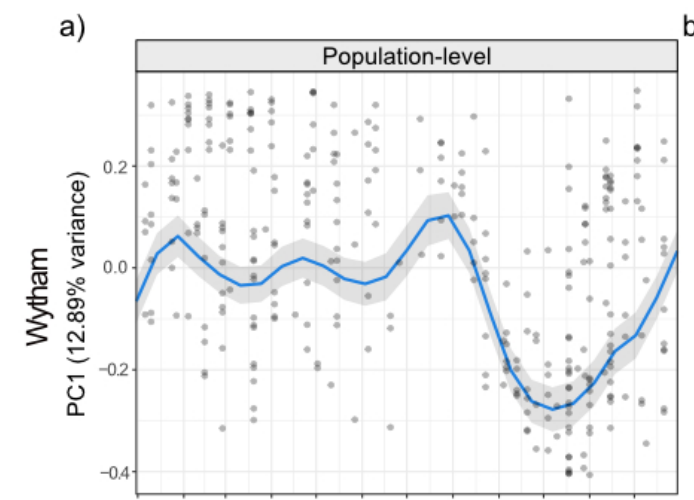

b)
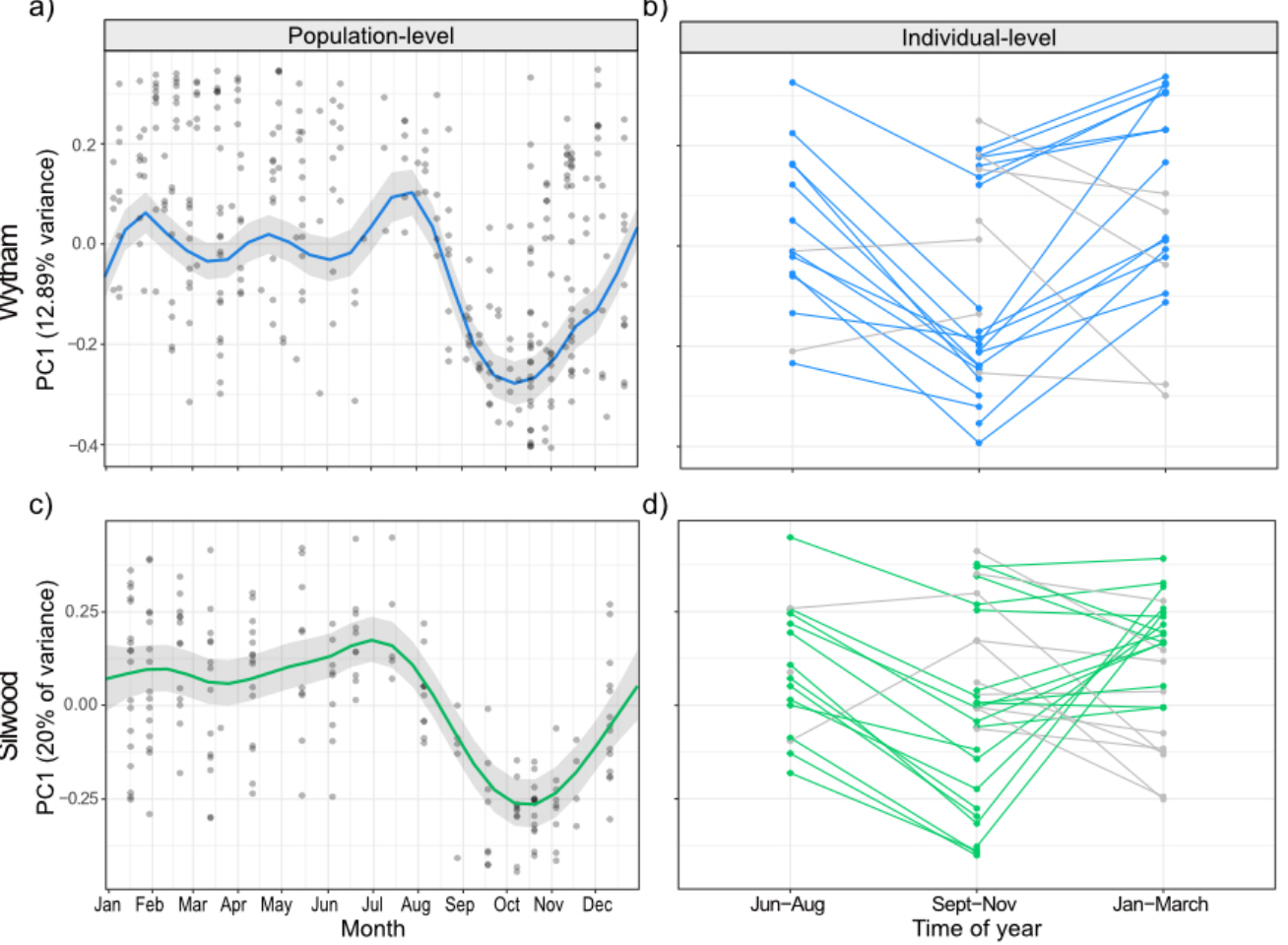

d)

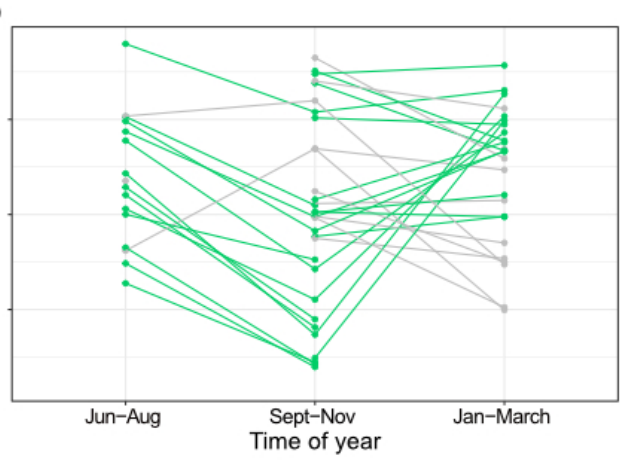

693 Figure 2. Seasonal restructuring of the wood mouse gut microbiota in two wild populations.

694 a) and c) Seasonal dynamics in population-level PC1 value (the first axis of a principal

695 coordinates analysis on Bray-Curtis dissimilarity values) in Wytham (top, blue) and Silwood

696 (bottom, green). Data from Wytham mice come from a 3-year period (Oct 2015-2018), while

697 Silwood mice were sampled for one year (November 2014-15). Predicted values and 95\%

698 confidence intervals for the smoothed day of the year from generalised additive mixed models

699 (GAMMs) are plotted. b) and d) Changes in PC1 within repeat-sampled individual mice typically

700 track the population-level seasonal shifts in both populations (coloured lines), with some

701 exceptions (grey lines). 


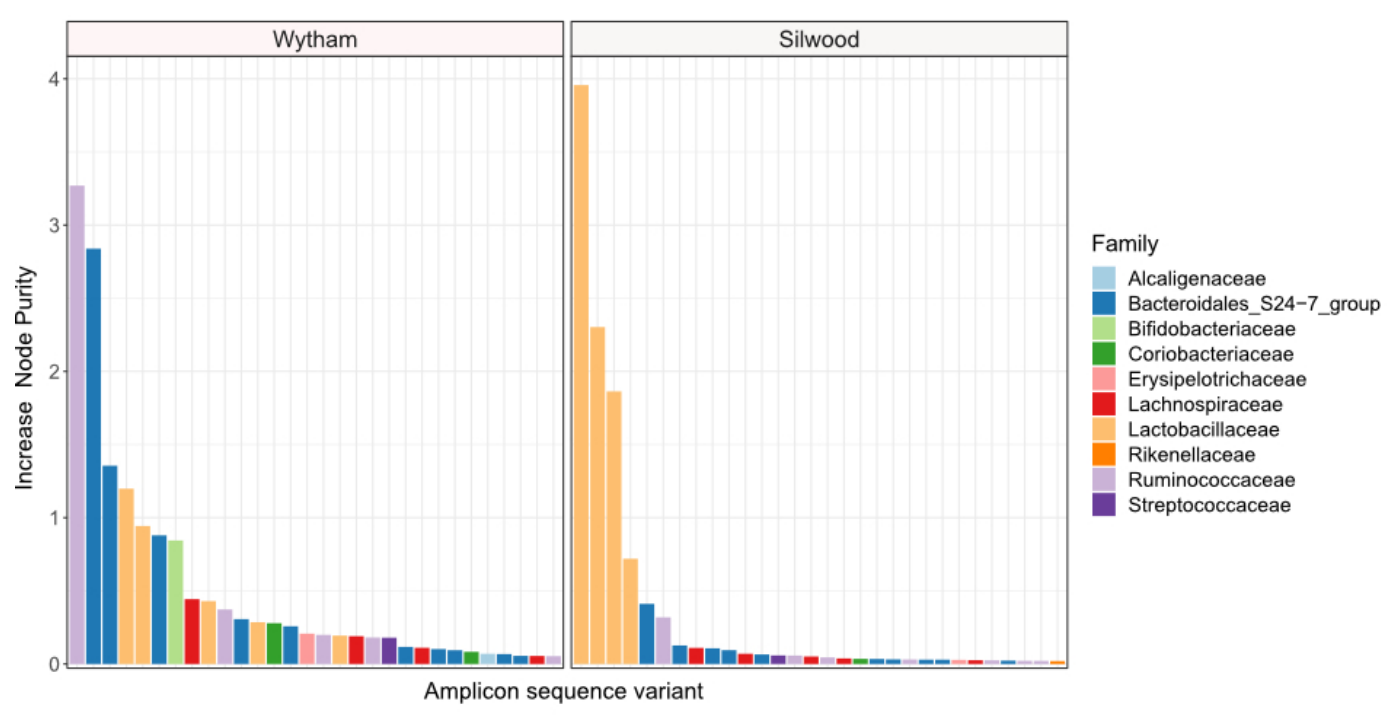

703 Figure 3. The importance of bacterial taxa in driving consistent seasonal patterns in PC1 (first

704 axis of a Bray-Curtis P(OA) in two wild wood mouse populations. Random forest regressions

705 (RFR) were used to identify bacterial amplicon sequence variants (ASVs) important for

706 predicting PC1, which has a strong seasonal signal, in each population. In Wytham (left), the

707 RFR could explain $92.11 \%$ of variation in PC1 using the relative abundance of ASVs as

708 predictors, while the Silwood the RFR could explain $93.31 \%$ of variation in PC1. The

709 'IncNodePurity' was used as a measure of feature importance in the models, and the top 30

710 strongest ASVs (highest IncNodePurity) are shown, with the bacterial family each ASV in

711 associated with in dicated by colour. 
Wytham

a)

b)

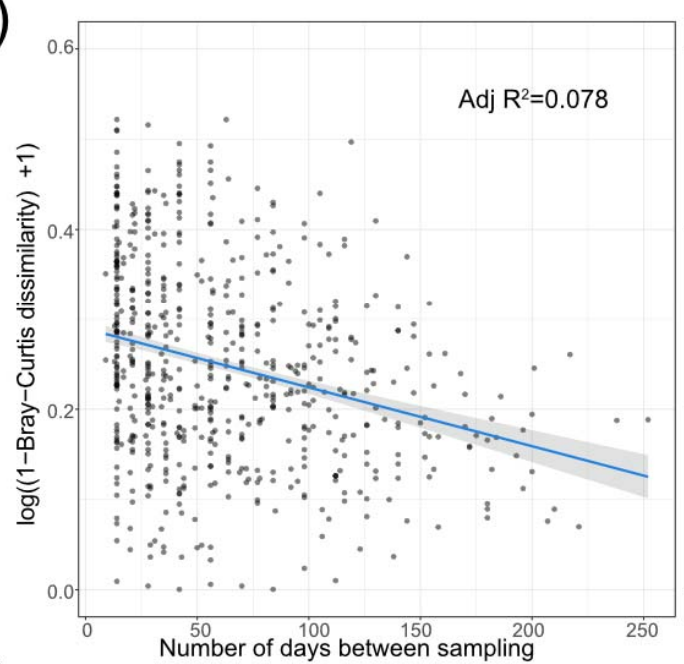

c)

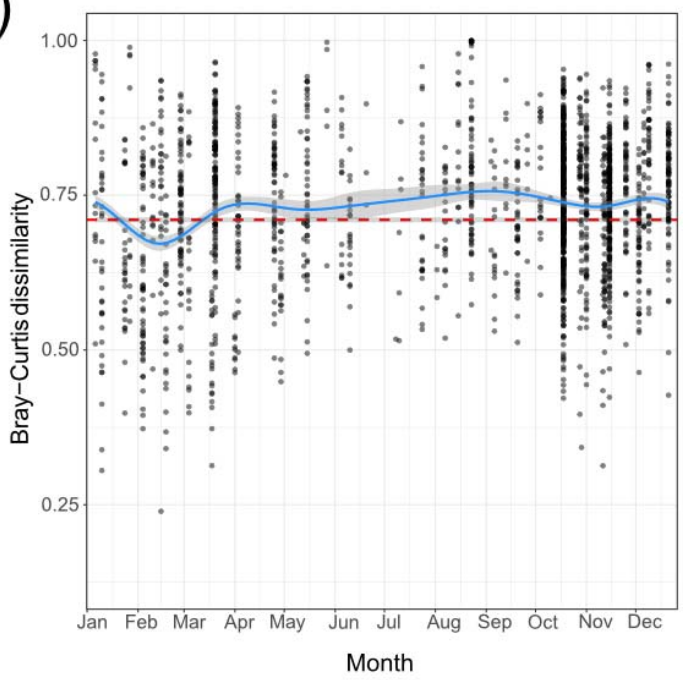

Silwood
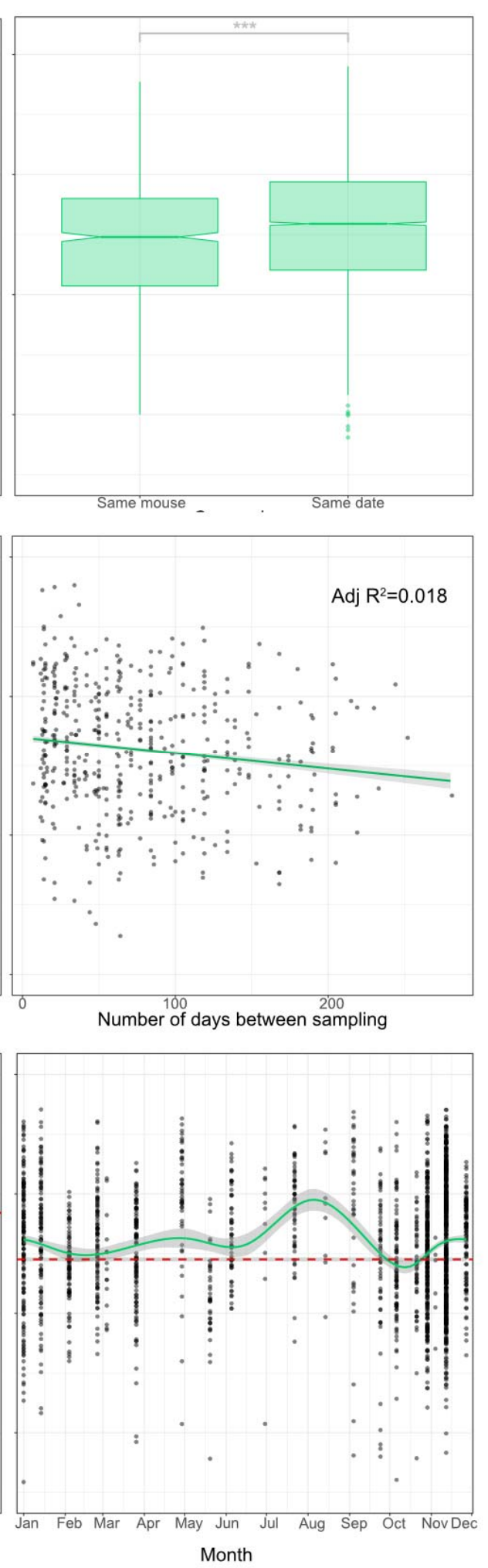
715 host at different times (Same mouse) and those taken from different hosts on the same day

716 (Same date) were used to compare intra- and inter-individual variation in Wytham (blue) and

717 Silwood (green) wild populations. Significant differences between groups were tested with

718 permutational Wilcoxon tests and are denoted by asterisks $(* * * ; p<0.001, * * ; p<0.01, *$;

$719 p<0.05$, NS; $p>0.05$ ) b) Intra-individual variation decays with sampling interval. Pairwise

720 community similarity (1- Bray-Curtis dissimilarity) between pairs of samples collected from the

721 same individual host, in mice that were captured three or more times in two separate

722 populations (Wytham; $n=277$ samples from 57 hosts and Silwood; $n=197$ samples from 39

723 hosts) are plotted against the number of days between the samples being collected.

724 Community similarity is log-transformed and the relationship is fitted using a log-linear model.

725 c) Inter-individual variation across the year was visualised by plotting the Bray-Curtis

726 dissimilarity between individuals per trapping session with a loess smoothing line for each wild

727 population. The mean ( \pm se) intra-individual Bray-Curtis dissimilarity per population is shown as

728 a dashed reference line in red.

729 Supporting Information

730 Supp_Mat_file1.docx 